\title{
Optical Activity of Graphene-Based Chiral Metasurface in THz Frequency Range
}

\author{
Maxim S. Masyukov ${ }^{1}$, Anna V. Vozianova ${ }^{1}$, Kseniia V. Gubaidullina $^{1}$, Alexander N. Grebenchukov ${ }^{1,2}$ \\ and Mikhail K. Khodzitsky ${ }^{1}$ \\ ${ }^{1}$ Terahertz Biomedicine Laboratory, ITMO University, Saint Petersburg, 199034 Russia \\ ${ }^{2}$ Ontoelectronics and Measurements Techniaues Laboratorv. Universitv of Oulu. Oulu. 90570 Finland
}

\begin{abstract}
In this paper the optical activity of two types of graphene-based chiral metasurfaces with two different variations of graphene inclusion positions was studied. The gammadion resonators of the unit cell were partly made of graphene. Three resonant frequencies of the metasurfaces in the frequency range of 0.1-0.5 THz were found. It is shown that the value of optical activity expressed in azimuth polarization rotation angle strongly depends on the chemical potential of graphene petals, as well as on the position of graphene inclusions.
\end{abstract}

\section{INTRODUCTION}

Q HIRALITY refers to a geometric property of an object when it doesn't have any mirror symmetry planes. This feature can be found in the structure of biological molecules, crystals, etc. The lack in mirror symmetry plane of molecules causes to many optical phenomena, such as optical rotation[1], linear or circular dichroism[2], polarizationsensitive absorption[3]. These effects were applied in development of metamaterials - a class of composite structured materials with specific properties, which usually cannot be found in nature [4]. Nowadays metamaterials are widely used in manipulation of terahertz wave properties as perfect absorbers[5], polarization sensitive components[6-9], band-pass filters and switchers [10-12]. In this research we propose graphene-based optical active metasurface with a chiral geometry of the unit cell. Unlike the other works devoted to this topic, all the substrate is not covered by graphene, namely only one half of chiral resonators are made of it.

\section{THE UNIT CELL DESIGN}

a)

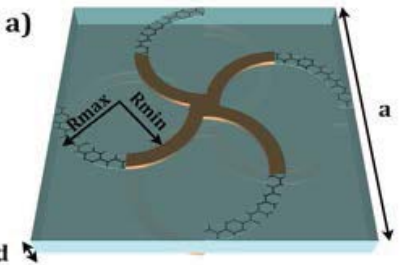

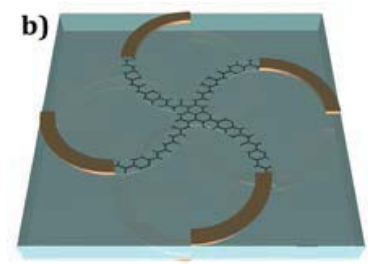

Fig. 1. Two types of chiral unit cell design considered in this paper.

Here we have studied two types of metasurface design shown in Fig. 1. The gammadion cross is made of 4 circular half-ring petals by their 90 -degree rotation around the normal to the unit cell plane at its central point. In both cases, gammadion resonators were precisely made of equal halves of graphene and gold, but in the first design (Fig. 1-a) the inner part of the resonators was made of gold and outer one was made of graphene, in the second case (Fig. 1-b) - vice versa. Geometrical properties of the unit cell are the following: the outer radius of the chiral resonator petals $\left(R_{\max }\right)$ is $150 \mu \mathrm{m}$, the inner one $\left(R_{\min }\right)$ is $125 \mu \mathrm{m}$, the thickness $(d)$ of the dielectric $(\varepsilon=2.1)$ substrate is $65 \mu \mathrm{m}$ and the unit cell period (a) is 600 $\mu \mathrm{m}$. The graphene conductivity was described by Kubo formula[13]. The substrate is made of TPX material with $\varepsilon=2.1$ at terahertz frequencies. The resonators which lie on the bottom side of the substrate have the same geometrical properties, but fully made of gold.

\section{NUMERICAL SIMULATION AND POLARIZING PROPERTIES CALCULATION APPROACH}

The numerical calculation of the radiation transmission was performed using CST Microwave Studio. Finite-elements method implemented in the frequency range of $0.1-0.5 \mathrm{THz}$. Periodic boundary conditions was used to simulate the infinite array of unit cells. Co- and cross-polarization spectra $\mathrm{T}_{\mathrm{xx}}$ and $\mathrm{T}_{\mathrm{xy}}$ were found to characterize the polarization state of the transmitted waves. After that, the transmission spectra for left handed ( $\left.\mathrm{T}_{--}\right)$and right-handed $\left(\mathrm{T}_{++}\right)$circularly polarized waves were found using the next formula:

$$
T_{ \pm \pm}=T_{x x} \pm i T_{x y}
$$

Azimuth polarization rotation angle $\theta$ can be found from phase difference of right-handed $(\mathrm{T}++)$ and left-handed (T--) circularly-polarized waves as[14]

$$
\theta=\frac{1}{2}\left[\arg \left(T_{++}\right)-\arg \left(T_{--}\right)\right] .
$$

This parameter $\theta$ characterizes the optical activity of the metasurface.

The scheme of numerical simulation is shown in Fig. 2.

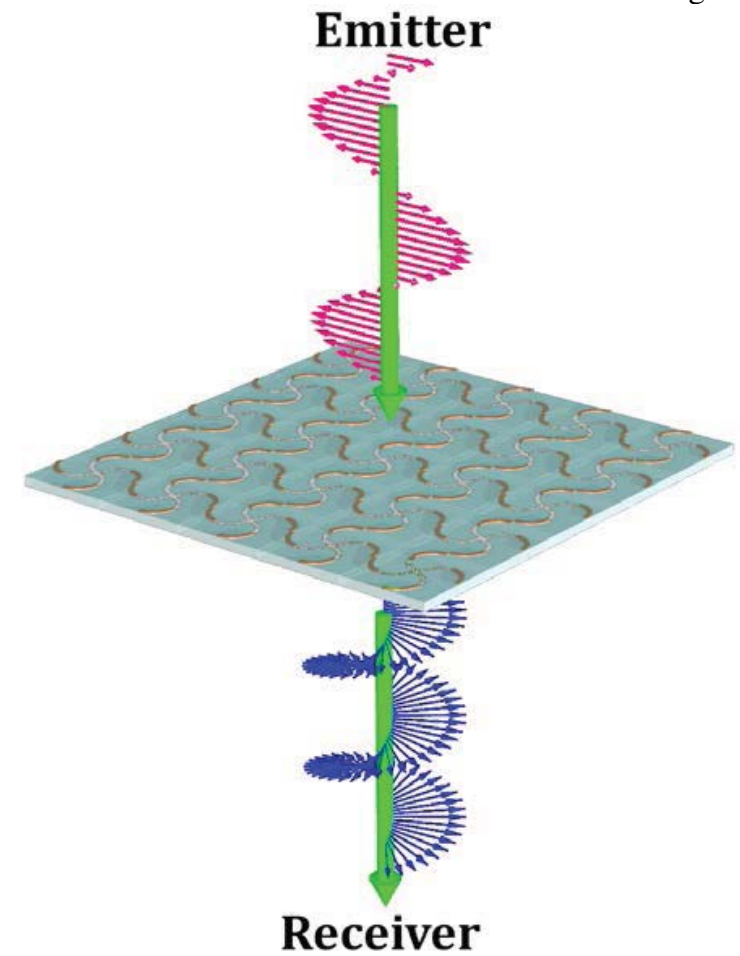

Fig. 2. The scheme of numerical simulation and polarization conversion. 


\section{RESULTS}

The spectra of optical activity for both types of the metasurface can be found in Fig. 3 .

As it can be seen from the azimuth angle spectra, due to the same geometry, there is almost no change in resonant frequencies of both types of metasurface. But in case when graphene part is at the center of the resonators (Fig. 1-b), we can find that the optical activity at $0.475 \mathrm{THz}$ increases very fast and the direction of rotation changes in contrast to the other case. Besides the metasurface with such design, unlike the other one, shows a high tunability at the frequency of 0.36 THz: azimuth polarization rotation angle decreases with increasing of graphene chemical potential. The metasurface with metal-based central parts of resonators reaches twice bigger maximum value of azimuth angle at the frequency of $0.25 \mathrm{THz}$ than one with graphene-based central parts of resonators.
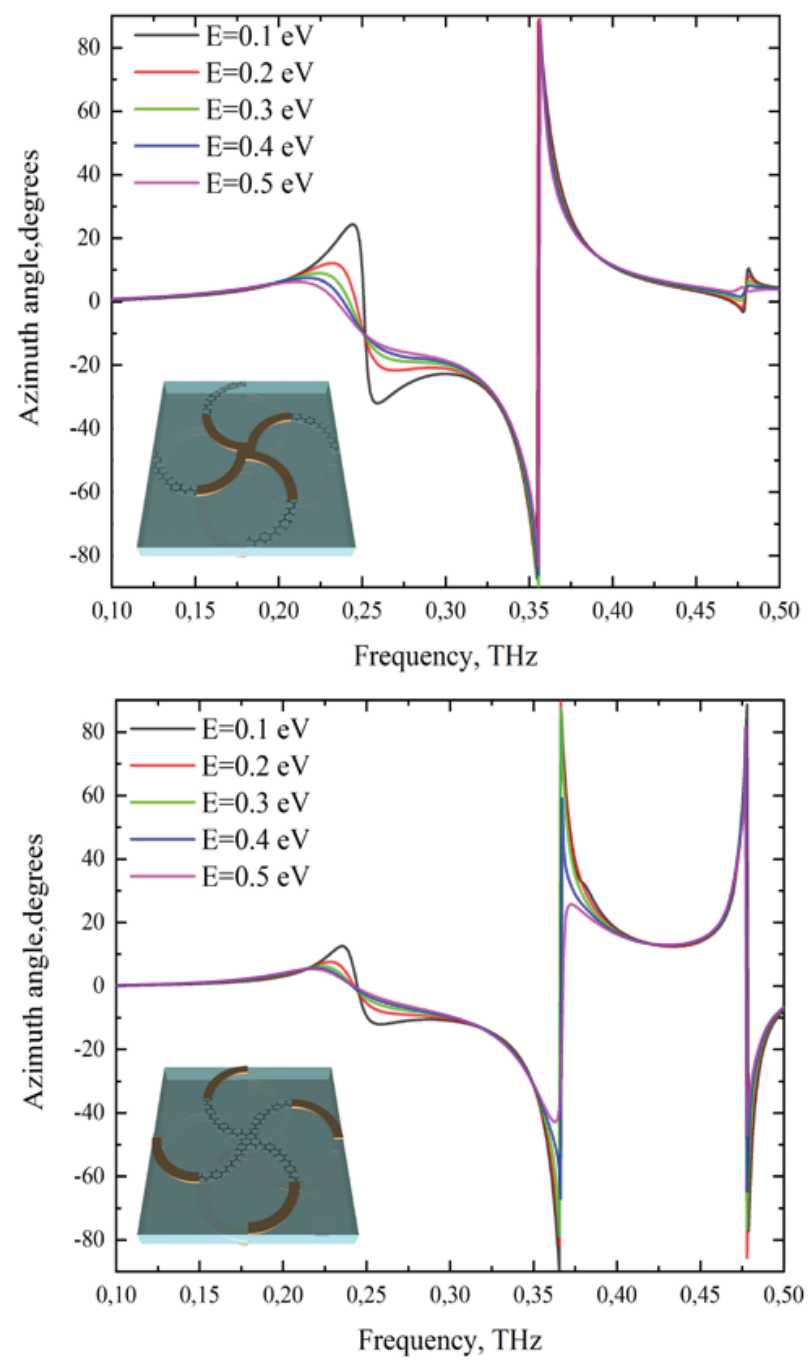

Fig. 3. Optical activity spectra for two types of the metasurface design

\section{SUMMARY.}

Optical activity of chiral metasurface with two different variations of graphene inclusion positions was studied in frequency range of 0.1-0.5 THz. In general, azimuth polarization rotation angle is changed at the frequencies of $v_{1}=0.25 \mathrm{THz}, v_{2}=0.36 \mathrm{THz}, v_{3}=0.475 \mathrm{THz}$ when chemical potential of graphene is varied, but the behavior of these resonances is different. The metasurface which central part of resonators made of graphene shows the most perceptible tunability. The results of this research may found its application in the development of waveplates or polarizationmodulating switchers for terahertz communications.

\section{ACKNOWLEDGEMENTS}

The reported study was funded by RFBR, project number 1931-90163

\section{REFERENCES}

[1] B. Mennucci, et al. "Polarizable continuum model (PCM) calculations of solvent effects on optical rotations of chiral molecules," The Journal of Physical Chemistry A, vol. 106 no. 252002, pp. 6102-6113, 2002

[2] T. B. Freedman et al, "Absolute configuration determination of chiral molecules in the solution state using vibrational circular dichroism," Chirality, vol.15, no. 9, pp. 743-758, 2003

[3] Y. Zhou et. al., "Optical coupling between chiral biomolecules and semiconductor nanoparticles: size-dependent circular dichroism absorption," Angewandte Chemie International Edition, vol. 50 no. 48, 2011

[4 ]Z. Wang, F. Cheng, T. Winsor and Y. Liu, "Optical chiral metamaterials: a review of the fundamentals, fabrication methods and applications," Nanotechnology, vol. 41, p 412001, November, 2016.

[5] D. Gomon, et al., "Influence of the geometric parameters of the electrical ring resonator metasurface on the performance of metamaterial absorbers for terahertz applications," Chinese Optics, vol.11, no.1, pp. 47-59, 2018

[6] E.Litvinov, P. Demchenko, E. Sheklanova, A. Vozianova, M. Khodzitsky, "Epsilon-near-zero copper-dielectric composite for terahertz frequency range," Proceedings of SPIE- The International Society for Optical Engineering, vol. 10719 p. 1071939, 2018

[7] M. Masyukov, A. Vozianova, A. Grebenchukov and M. Khodzitsky, "Polarizing properties of chiral metasurface based on gammadion crosses with different geometry in THz frequency range," Proceedings of SPIE- The International Society for Optical Engineering, vol. 10343 p. 1034338, 2017

[8] A.N. Grebenchukov, et al." Faraday effect control in graphene-dielectric structure by optical pumping," Journal of Magnetism and Magnetic Materials, vol. 472 pp. $25-28,2019$

[9] S. Y Korolenko. et al "Simulation of polarizer based on chiral medium for terahertz frequency range," Journal of Physics: Conference Series, vol. 735 no. 1, p.012089, 2016

[10] V. Yu. Soboleva et al., "Development of narrow bandpass filters based on cross cavities for the terahertz frequency range," Journal of Optical Technology, vol. 84, no. 8, pp. 521-524, 2017.

[11] A.N. Grebenchukov, et al., "Optically controlled narrowband terahertz switcher based on graphene," Chinese optics, vol.11, no.2, pp. 2095-1531, 2018.

[12] S. Smirnov et. al. "Optically controlled dielectric properties of singlewalled carbon nanotubes for terahertz wave applications," Nanoscale, vol. 10 no. 26, pp. 12291-12296, 2018.

[13] L. A. Falkovsky et. al., "Optical properties of graphene," Journal of Physics: Conference Series, vol. 129. no. 1, 2008

[14] G. Kenanakis, E.N. Economou, C.M. Soukoulis and M. Kafesaki, "Controlling $\mathrm{THz}$ and far-IR waves with chiral and bianisotropic metamaterials," EPJ Applied Metamaterials, vol. 2, pp. 15, 2015. 